\author{
AVALIAÇÃO DE TOXICIDADE, COMPOSIÇÃO QUÍMICA \\ E SENSORIAL DA SARCOCÓRNIA AMBIGUA: USO EM \\ POPULAÇÃO HIPERTENSA
}

\author{
EVALUATION OF TOXICITY, CHEMICAL COMPOSITION AND \\ SENSORY OF SARCOCORNIA AMBIGUA: USE IN \\ HYPERTENSIVE POPULATION
}

\begin{abstract}
Amanda Rosa Coelho'
Discente do Curso de Medicina, Universidade Alto Vale do Rio do Peixe - UNIARP -

Caçador/SC, e-mail: a.amandac@hotmail.com
\end{abstract}

Talize Foppa ${ }^{2}$

Mestre e docente do Curso de Medicina, Universidade Alto

Vale do Rio do Peixe - UNIARP

- Caçador/ SC, e-mail:

talize@uniarp.edu.br

\section{Andressa Ana}

Ansiliero $^{3}$

Discente do Curso de pós

graduação em Ecossistemas

Agrícolas e Naturais,

Universidade Federal de Santa

Catarina - UFSC -

Curitibanos/SC, e-mail:

andressa.ansiliero@gmail.com

\section{RESUMO}

A Sarcocornia ambigua é uma halófita (tolerante ao sal) que cresce em áreas com elevadas concentrações salinas, capaz de absorver os nutrientes e minerais do ambiente em que vive. Devido à sua riqueza de compostos bioativos, tornase uma alternativa promissora na manutenção da pressão arterial, através da substituição do sal nos alimentos. Sendo assim, o presente trabalho teve como objetivo avaliar a toxicidade in vitro da Sarcocornia ambígua, avaliar a influência da temperatura de cozimento dos alimentos na quantificação dos compostos químicos: fenóis, ácido ascórbico, betacaroteno e minerais e analisar sensorialmente, em população de hipertensos e a sua aceitação como substituinte do sal de cozinha em um produto alimentício. Diante disso, não foram encontrados sinais tóxicos da planta e não houve diferenças significativas entre os tratamentos, portanto, não havendo perda da qualidade nutricional do sal verde após o preparo de produtos alimentícios. Além disso, foi possível determinar altos níveis de fenóis, ácido ascórbico e minerais, com predomínio de potássio, sódio, magnésio e cálcio. No que se refere à análise sensorial, o produto alimentício temperado com o sal verde teve aceitação significativa. Sendo assim, a Sarcocornia ambigua demonstrou-se como uma fonte vegetal de elevada qualidade nutricional, agradável ao paladar e uma alternativa benéfica como coadjuvante no tratamento da hipertensão arterial.

PALAVRAS-CHAVE: Halófita. Hipertensão. Análise sensorial.

\section{ABSTRACT}

Sarcocornia ambigua is a halophyte (salt tolerant) that grows in areas with high salt concentrations, capable of absorbing nutrients and minerals from the environment in which it lives. Due to its wealth of bioactive compounds, it becomes a promising alternative in maintaining blood pressure, by replacing salt in food. Therefore, the present study aimed to evaluate the in vitro toxicity of Sarcocornia ambigua, to evaluate the influence of the cooking temperature of the food in the quantification of chemical compounds: phenols, ascorbic acid, betacarotene and minerals and to analyze sensorially in a hypertensive population and its acceptance as a substitute for table salt in a food product. Therefore, no 
toxic signs of the plant were found and there were no significant differences between treatments, therefore, there was no loss of the nutritional quality of green salt after the preparation of food products. In addition, it was possible to determine high levels of phenols, ascorbic acid and minerals, with a predominance of potassium, sodium, magnesium and calcium. With regard to sensory analysis, the food product seasoned with green salt had significant acceptance. Therefore, Sarcocornia ambigua has shown itself to be a vegetable source of high nutritional quality, pleasant to the taste and a beneficial alternative as an adjunct in the treatment of arterial hypertension.

KEYWORDS: Halophyte. Hypertension. Sensory analysis.

\section{INTRODUÇÃO}

A hipertensão arterial destaca-se como uma doença de relevância epidemiológica devido a sua alta prevalência e por ser um dos principais fatores de risco para o desenvolvimento de doenças cardiovasculares (SILVA et al., 2013; MENDES; MORAES; GOMES, 2014). Segundo Girotto et al. (2013), uns dos maiores desafios no combate à hipertensão arterial se deve à falta de adesão ao tratamento farmacológico e da adoção de um estilo de vida adequado. E uma das principais e mais baratas estratégias de controle da pressão arterial é a mudança dos hábitos alimentares, especialmente a redução do consumo de sal na dieta.

O sal é encontrado nos alimentos na forma de cloreto de sódio (39,3\% de sódio e $60,7 \%$ de cloreto) e é responsável por $90 \%$ do sódio da alimentação (DOS SANTOS et al., 2015). A alta ingestão desse elemento induz ao endurecimento endotelial através da diminuição da liberação do vasodilatador óxido nítrico (NO), causando disfunção vascular e resultando na manifestação e/ou agravamento da hipertensão arterial (PANTH et al., 2016).

Segundo as VII Diretrizes Brasileiras de Hipertensão Arterial, o limite de consumo diário de sódio associado à diminuição da pressão arterial é de $2,0 \mathrm{~g}$, no entanto, o consumo do brasileiro pode chegar até 11,4 g/dia (BRASIL, 2016). Por isso, recomenda-se reduzir a quantidade de sal na dieta, substituindo por outras substâncias que ressaltem o sabor dos alimentos, mas não influenciem na pressão arterial, como o vinagre, limão, cebola, azeite de oliva, pimenta, salsa, cebolinha, alho, coentro, manjericão, louro, páprica, orégano e alecrim (BEZERRA, 2009; DE OLIVEIRA; MOREIRA, 2016). Os temperos e especiarias, além de tornarem os alimentos mais atrativos, fornecem características organolépticas agradáveis, ajudam na diminuição do uso de sal e também agregam propriedades nutricionais e terapêuticas (BEZERRA, 2009).

Atualmente, vem crescendo o interesse em pesquisas direcionadas ao estudo e desenvolvimento de substâncias naturais envolvidas na prevenção e no controle da hipertensão arterial. Em destaque, está a Sarcocornia ambigua, halófita da família Amaranthaceae, capaz de tolerar ambientes com elevadas concentrações salinas, baixa disponibilidade de matéria orgânica, submersão periódica, drenagem rápida da água e luz solar direta e intensa (BERTIN et al., 2014).

A sobrevivência sob essas condições estressantes permitiu à Sarcocornia ambigua desenvolver mecanismos de resposta fisiológica e bioquímica, que concedem valor nutricional e variedade de compostos bioativos. Dessa forma, a 
planta torna-se uma alternativa para a substituição do sal na dieta, com o objetivo de manter a saúde cardiovascular (PANTH et al., 2016).

O equilíbrio osmótico, uma das principais adaptações ao estresse salino da planta, é responsável por absorver controladamente os sais do ambiente pelo sistema radicular e acumular em concentração igual à da água do mar, sem nenhum agravo para a planta (PEREIRA et al., 2012; VENTURA; SAGI, 2013; BERTIN et al., 2014). Por conseguinte, o acúmulo de sais nas células é equilibrado pela síntese de solutos orgânicos, como os açúcares e aminoácidos. Esses compostos, caracterizados como osmoprotetores, são capazes de manter o gradiente de potencial da água favorável e proteger as estruturas celulares (MANOUSAKI; KALOGERAKIS, 2010; PEREIRA et al., 2012).

As plantas também desenvolveram um mecanismo para neutralizar os danos oxidativos causados pela formação de espécies reativas de oxigênio (ROS). Desta forma, ocorre a síntese de enzimas, como a catalase, superóxido dismutase e glutationa redutase e compostos não enzimáticos como o ascorbato, tocoferol e os fenóis, que contribuem para a atividade antioxidante da planta (VENTURA; SAGI, 2013; BERTIN et al., 2014; PINHEIRO, 2015).

A família Amaranthaceae possui dois grandes gêneros, Salicornia e Sarcocornia, ambas utilizadas historicamente na alimentação, principalmente no mercado europeu, como vegetal in natura semelhante ao aspargo verde ou como especiaria, devido ao sabor salgado e elevado valor nutritivo (PEREIRA et al., 2012; GARGOURI et al., 2013; PINHEIRO et al., 2017). Essas halófitas são caracterizadas pela riqueza em metabólitos nutricionalmente benéficos, como os fenóis, ácidos graxos, ácido ascórbico, betacaroteno e minerais, responsáveis pelas propriedades terapêuticas da planta. Contudo, a quantidade desses compostos pode variar de acordo com a espécie, sazonalidade, localização geográfica, clima, tipo de solo, quantidade de água e nutrientes disponíveis e presença de agentes estressores, como a luz (GARGOURI et al., 2013; BERTIN et al., 2014; PINHEIRO, 2015; GUNNING, 2016).

No Brasil, encontra-se a espécie Sarcocornia ambigua, anteriormente conhecida como Salicornia gaudichaudiana. Adaptada a viver nos mangues, com morfologia simples, brotos suculentos, sem folhas e com um ciclo de vida perene (IZEPPI, 2011; BERTIN et al., 2014; PINHEIRO et al., 2017), a Sarcocornia ambigua, assim como a Salicornia ramosissima, quando seca e triturada, é transformada em uma especiaria, o sal verde, que pode ser utilizada como tempero (JULIÃO, 2013).

Vários estudos avaliaram as potenciais propriedades terapêuticas da Salicornia e da Sarcocornia destacando, a ação na diminuição do peso corporal e nos perfis lipídicos de ratos obesos, no tratamento de osteoporose, a atividade antioxidante e despigmentante da pele, no controle da diabetes através do aumento do glicogênio hepático e muscular, na melhora da função intestinal e a ação protetora vascular (KIM; JUN; KIM; 2015; KARADENIZ et al., 2014; BERTIN et al., 2014; PATEL, 2016; KIM; KIM; LEE, 2014; PANTH et al., 2016). Porém, não há estudo publicado avaliando se a temperatura de cozimento afeta as propriedades nutricionais e terapêuticas das halófitas, especialmente da Sarcocornia ambigua. Além disso, há necessidade em verificar a aceitação sensorial para que a mesma possa ser comercialmente viável na população em que se pretende inserir.

Desse modo, o objetivo desse trabalho foi avaliar a toxicidade in vitro da Sarcocornia ambigua, avaliar a influência da temperatura de cozimento dos alimentos na quantificação dos compostos químicos e analisar sensorialmente, 
em população de hipertensos, a sua aceitação como substituinte do sal de cozinha em um produto alimentício.

\section{MATERIAL E MÉTODOS}

\section{Material vegetal e preparo da amostra}

O material vegetal foi coletado no Laboratório de Camarões Marinhos da Universidade Federal de Santa Catarina, com depósito no herbário Dr. Roberto Miguel Klein do Departamento de Botânica da Universidade Regional de Blumenau (FURB) sob $\mathrm{n}$ ㄴ 41346. As partes aéreas da planta foram submetidas ao controle da umidade, até atingir peso constante, segundo método proposto pela Farmacopeia Brasileira (2010). Após, foram trituradas em liquidificador, até formar uma farinha fina.

\section{Toxicidade}

Para a determinação da toxicidade utilizou-se o sistema Allium cepa, aplicando água como controle negativo e CuSO4 (6ppm) como controle positivo. A amostra foi preparada na forma de infusão, com $2 \mathrm{~g}$ da planta em $150 \mathrm{~mL}$ de água e deixada em contato com as raízes da cebola por 72 horas. A análise foi realizada através da medição do comprimento das raízes das cebolas e a porcentagem de inibição do crescimento radicular (KRUGER, 2009 adaptado).

\section{Tratamentos térmicos}

Para realizar os tratamentos térmicos, utilizou-se de método gravimétrico com temperaturas pré-determinadas (Tabela 1) por 20 minutos (CASTRO et al., 2017 adaptado).

Tabela 1 - Tratamentos térmicos para avaliação da influência da temperatura na composição química da Sarcocornia ambigua

\begin{tabular}{cc} 
Amostra & Temperatura \\
\hline I & $40^{\circ} \mathrm{C}$ \\
II & $60^{\circ} \mathrm{C}$ \\
III & $80^{\circ} \mathrm{C}$ \\
IV & $100^{\circ} \mathrm{C}$ \\
\hline & Fonte: Castro et al. adaptado (2017).
\end{tabular}

\section{Composição Química}

Realizou-se a dosagem do conteúdo fenólico pelo método colorimétrico de FolinCiocalteau, utilizando curva padrão de ácido gálico com concentrações de 25 $\mu \mathrm{g} / \mathrm{mL}, 50 \mu \mathrm{g} / \mathrm{mL}, 75 \mu \mathrm{g} / \mathrm{mL}, 100 \mu \mathrm{g} / \mathrm{mL}$ e $125 \mu \mathrm{g} / \mathrm{mL}$, e os resultados foram expressos em equivalente de ácido gálico (IVANOVA et al., 2006 adaptado). 
A dosagem de ácido ascórbico foi realizada de acordo com Abreu e Faria (2007) e os resultados expressos em $\mathrm{mg} / 100 \mathrm{~g}$ da amostra.

A dosagem de betacaroteno foi realizada a partir da metodologia de Rocha et al. (2013) por espectrofotometria e os resultados foram expressos por $\mathrm{mg} / 100 \mathrm{~g} \mathrm{da}$ amostra.

E a dosagem de minerais foi realizada por espectrofotometria de absorção atômica e os resultados foram expressos em $\mathrm{g} / 100 \mathrm{~g}$ e $\mathrm{mg} / 100 \mathrm{~g}$ da amostra (SCHVEITZER; SUZUKI, 2013).

\section{Análise sensorial}

A escolha do produto alimentício para o teste sensorial foi a batata frita congelada, frita no momento do teste, temperada com o sal verde. Para a análise sensorial, participaram 40 julgadores não treinados, hipertensos, participantes do Grupo de Hipertensos e Diabéticos de um posto de saúde do município de Caçador/SC. Foram servidos aproximadamente $5 \mathrm{~g}$ de amostra, em pratos descartáveis e os provadores foram instruídos a realizar a lavagem da cavidade oral com água filtrada antes do teste. Para a avaliação foi utilizado o teste de aceitação com escala hedônica de 9 pontos, expressando o grau de gostar ou desgostar do produto, variando de 1-desgostei extremamente a 9-gostei extremamente; e o teste de atitude ou intenção com escala de 7 pontos, expressando a vontade de consumir o produto, variando de 1-nunca comeria a 7-comeria sempre (GASTALDON et al., 2007 adaptado).

\section{Análise estatística}

Os dados foram realizados em triplicatas e avaliados no Microsoft Excel através de análise de variância ANOVA e teste Duncan, com nível de significância 5\%. O projeto foi submetido ao comitê de ética de humanos na UNIARP Universidade Alto Vale do Rio do Peixe, sob número 69045317.2.0000.5593.

\section{RESULTADO E DISCUSSÃO}

\section{Toxicidade}

O estudo investigou a potencial ação tóxica da Sarcocornia ambigua através da avaliação do comprimento das raízes do bulbo das cebolas e da porcentagem de inibição do crescimento radicular pelo método Allium cepa. As raízes de Allium cepa expostas à infusão da Sarcocornia ambigua tiveram o desenvolvimento de suas raízes comparadas aos controles, como mostra a Tabela 2. 
Tabela 2- Avaliação da toxicidade da Sarcocornia ambigua através do comprimento médios das raízes de Allium cepa e da porcentagem de inibição do crescimento radicular.

\begin{tabular}{|c|c|c|}
\hline Tratamento & $\begin{array}{l}\text { Comprimento médio das raizes } \\
\qquad(\mathrm{cm})\end{array}$ & $\begin{array}{l}\text { Porcentagem de inibição do } \\
\text { crescimento radicular }\end{array}$ \\
\hline Controle Negativo & $0,66+-0,06^{* a}$ & $N A^{* *}$ \\
\hline Controle Positivo & $0,13+-0,05^{* b}$ & $80.3 \%$ \\
\hline Sarcocornia ambigua & $0,57+-0,03^{\star a}$ & $13,6 \%$ \\
\hline
\end{tabular}

Fonte: As autoras (2017).

Verifica-se que não houve diferença significativa no crescimento radicular da planta quando comparado ao controle negativo $(p>0,05)$. Na análise da toxicidade foram obtidas reduções no crescimento radicular de $13,6 \%$ para a Sarcocornia ambigua, já no controle positivo de toxicidade, a redução foi de $80,3 \%$. Sugerindo assim, que a Sarcocornia ambigua não apresentou risco tóxico em relação à inibição do ciclo celular.

Não foram encontrados estudos que identificassem a toxicidade in vitro das halófitas do gênero Sarcocornia e Salicornia. Entretanto, Lee (2016) analisou a toxicidade da Salicornia herbacea in vivo e constatou não haver sinais clínicos anormais, alterações patológicas, bioquímicas e nem alterações no peso dos animais que indicassem a presença de toxicidade. Todavia, o extrato foi capaz de diminuir significativamente os níveis de colesterol total e de triglicérides, indicando que além de apresentar biossegurança estável, exibe ação em potencial para o tratamento de hiperlipidemias.

\section{Análise da composição química}

Os resultados obtidos na avaliação dos fenóis, ácido ascórbico e betacaroteno da Sarcocornia ambigua submetida aos tratamentos térmicos estão representados na Tabela 3.

Tabela 3 - Avaliação dos fenóis, ácido ascórbico e betacaroteno da Sarcocornia ambigua exposta aos tratamentos térmicos

\begin{tabular}{|c|c|c|c|c|}
\hline $\begin{array}{l}\text { Compostos } \\
\text { Quimicos }\end{array}$ & $\begin{array}{c}\mathrm{I}^{\star} \\
\text { Média } \pm \mathrm{DP}^{* *}\end{array}$ & $\begin{array}{c}I^{*} \\
\text { Média } \pm \mathrm{DP}^{* *}\end{array}$ & $\begin{array}{c}\text { III }^{*} \\
\text { Média } \pm \mathrm{DP}^{\star *}\end{array}$ & $\begin{array}{c}\text { IV }^{*} \\
\text { Média } \pm \mathrm{DP}^{* *}\end{array}$ \\
\hline $\begin{array}{c}\text { Fenóis } \\
\left.\text { (mg EAG } 100 \mathrm{~g}^{-1}\right)\end{array}$ & $48,70 \pm 0,19^{a}$ & $51,15 \pm 0,18^{a}$ & $47,00 \pm 0,51^{a}$ & $44,90 \pm 0,30^{6}$ \\
\hline $\begin{array}{c}\text { Acido ascórbico } \\
\text { (mq/100g da amostra) }\end{array}$ & $0,45 \pm 0,02^{3}$ & $0,44 \pm 0,02^{\mathrm{a}}$ & $0,42 \pm 0,01^{3}$ & $0,44 \pm 0,03^{a}$ \\
\hline $\begin{array}{c}\text { Betacaroteno } \\
\text { (mg/100g da amostra) }\end{array}$ & $0,26 \pm 0,01^{3}$ & $0,23 \pm 0,02^{3}$ & $0,25 \pm 0,01^{3}$ & $0,32 \pm 0,02^{a}$ \\
\hline
\end{tabular}

${ }^{*}$ Amostras expostas aos tratamentos térmicos $\left(1-40^{\circ} \mathrm{C}\right.$; II- $60^{\circ} \mathrm{C}$; III- $80^{\circ} \mathrm{C}$ e IV- $\left.100^{\circ} \mathrm{C}\right)$

** Desvio padrão das médias

a-b Diferentes letras entre os tratamentos representam diferenças significativas em cada linha $(p<0,05)$ Fonte: As autoras (2017).

A dosagem dos fenóis apresentou valores comparáveis aos obtidos por Pinheiro (2015) na Sarcocornia ambigua com 41,34 mg EAG 100g-1, mas inferiores aos obtidos por Julião (2013) na Salicornia ramosissima, com 500 mg EAG 100g-1. Ainda assim, os valores obtidos estão próximos aos encontrados em outros vegetais ricos em fenóis, como aspargo, pimentão verde, cebola e tomate ( $>50$ mg EAG 100g-1) (PINHEIRO, 2015).

Em relação ao ácido ascórbico, os resultados são similares ao encontrado por Riquelme et al. (2016) na Sarcocornia neei, com 0,55 mg/100g, e inferiores ao 
encontrado por Julião (2013) na Salicornia ramosissima, com 0,86 mg/100g. Além disso, os resultados obtidos demonstraram-se superiores aos encontrados em outros vegetais, como o espinafre, variando de 0,22 a 0,31 mg/100 (RIQUELME et al., 2016).

O teor de betacaroteno obtido foi baixo, visto que Riquelme et al. (2016) encontrou 0,46 mg/100g no extrato da Sarcocornia neei, enquanto Julião (2013) encontrou $0,65 \mathrm{mg} / 100 \mathrm{~g}$ na Salicornia ramosissima. Buscando esclarecer variações encontradas na dosagem desse composto, Agawu (2012) relacionou a diminuição do conteúdo de betacaroteno com o aumento no nível de salinidade no ambiente. Visto que a coleta do material vegetal ocorreu em período de pouca chuva, sugere-se que houve influência nos valores de betacaroteno devido à diminuição da salinidade do ambiente. Já comparado com amostras de cenoura in natura, uma das principais fontes de betacaroteno, Silva et al (2016) encontrou a presença de 2,0 mg/100g, valor acima ao encontrado na Sarcocornia ambigua. Dos minerais analisados, verifica-se na Tabela 4, que a planta obteve predomínio dos minerais potássio e sódio, seguido de magnésio e cálcio. Resultado equivalente ao encontrado nas halófitas Salicornia herbacea e Salicornia europaea (BERTIN et al., 2014).

Tabela 4- Avaliação dos minerais da Sarcocomia ambigua exposta aos tratamentos térmicos

\begin{tabular}{|c|c|c|c|c|}
\hline Minerais & ${\text { Média } \pm \mathrm{DP}^{\star \star \star}}^{\star}$ & $\begin{array}{c}\|^{*} \\
\text { Média } \pm \mathrm{DP}^{\star \star}\end{array}$ & $\begin{array}{c}\text { III* }^{*} \\
\text { Média } \pm \mathrm{DP}^{\star \star}\end{array}$ & $\begin{array}{c}\text { IV }^{*} \\
\text { Média } \pm \mathrm{DP}^{\star \star}\end{array}$ \\
\hline $\begin{array}{c}\text { Sódio } \\
\text { (g/100g da amostra) }\end{array}$ & $10,8 \pm 0,03^{a}$ & $11,5 \pm 0,26^{a}$ & $11,0 \pm 0,01^{a}$ & $11,5 \pm 0,47^{3}$ \\
\hline $\begin{array}{c}\text { Potássio } \\
\text { ( } \mathrm{g} / 100 \mathrm{~g} \mathrm{da} \text { amostra) }\end{array}$ & $21,4 \pm 0,1^{a}$ & $26,0 \pm 0,02^{\mathrm{a}}$ & $22,0 \pm 0,17^{\mathrm{a}}$ & $27,8 \pm 0,5^{\mathrm{a}}$ \\
\hline $\begin{array}{c}\text { Cálcio } \\
\text { (g/100g da amostra) }\end{array}$ & $0,4 \pm 0,02^{\mathrm{a}}$ & $0,4 \pm 0,15^{a}$ & $0,9 \pm 0,4^{a}$ & $0,9 \pm 0,01^{\mathrm{a}}$ \\
\hline $\begin{array}{c}\text { Magnésio } \\
\text { (g/100g da amostra) }\end{array}$ & $7,3 \pm 0,8^{a}$ & $8,0 \pm 0,02^{\mathrm{a}}$ & $8,0 \pm 0,21^{a}$ & $5,2 \pm 0,9^{b}$ \\
\hline $\begin{array}{c}\text { Ferro } \\
\text { (mg/100g da amostra) }\end{array}$ & $44,0 \pm 0,5^{a}$ & $38,0 \pm 1,1^{\mathrm{a}}$ & $52,0 \pm 0,07^{a}$ & $46,0 \pm 0,01^{3}$ \\
\hline $\begin{array}{c}\text { Žinco } \\
\text { (mg/100g da amostra) }\end{array}$ & $30,0 \pm 0,19^{a}$ & $37,0 \pm 0,64^{a}$ & $32,0 \pm 0,01^{a}$ & $36,0 \pm 0,07^{a}$ \\
\hline $\begin{array}{c}\text { Čobre } \\
\text { (mg/100g da amostra) }\end{array}$ & $3,0 \pm 0,01^{a}$ & $4,0 \pm 0,52^{\mathrm{a}}$ & $3,0 \pm 0,09^{\mathrm{a}}$ & $4,0 \pm 0,3^{3}$ \\
\hline
\end{tabular}

O teor de sódio obtido está próximo ao encontrado na Sarcocornia neei $(9,04$ $\mathrm{g} / 100 \mathrm{~g})$ e na Salicornia europeae $(12,9 \mathrm{~g} / 100 \mathrm{~g})$ e discretamente inferior à Salicornia ramosissima $(18,19 \mathrm{~g} / 100 \mathrm{~g})$ e à Sarcocornia perennis $(16 \mathrm{~g} / 100 \mathrm{~g})$ (JULIÃO, 2013; RIQUELME et al., 2016). Já o teor de potássio demonstrou-se superior aos encontrados na Salicornia ramosissima $(1,06 \mathrm{~g} / 100 \mathrm{~g})$, na Sarcocornia neei $(1,79 \mathrm{~g} / 100 \mathrm{~g})$, na Sarcocornia perennis $(1,11 \mathrm{~g} / 100 \mathrm{~g})$ e na Salicornia europeae (1,35 g/100g) (JULIÃO, 2013; RIQUELME et al., 2016).

Visto que obtiveram-se dosagens maiores de potássio do que de sódio, estudos indicam que enquanto o consumo de sódio está associado com a elevação da pressão arterial e doenças cardiovasculares, o consumo de potássio, por sua vez, pode diminuir o risco dessas doenças (ALVES, 2014). O potássio provoca a diminuição da concentração de sódio intracelular através da redução da atividade adrenérgica neural e dos níveis da renina plasmática, da inibição da formação de radicais livres e do aumento da atividade da enzima $\mathrm{Na+} / \mathrm{K}_{+}-$ ATPase, trazendo benefícios para a saúde dos hipertensos. Dessa forma, a 
Sarcocornia ambigua demonstra-se com potencial para atuar como coadjuvante no tratamento da hipertensão, por restringir o consumo de sódio nos alimentos e por possuir grandes quantidades de potássio (TOMAZONI; SIVIERO, 2009; RODRIGUES et al., 2015).

Em relação aos demais minerais, a Sarcocornia ambigua exibiu na dosagem de cálcio resultados semelhantes às halófitas Sarcocornia neei $(0,58 \mathrm{~g} / 100 \mathrm{~g})$, Salicornia europeae $(0,38 \mathrm{~g} / 100 \mathrm{~g})$ e Sarcocornia perennis $(0,31 \mathrm{~g} / 100 \mathrm{~g})$ e superiores à Salicornia ramosissima $(0,27 \mathrm{~g} / 100 \mathrm{~g})$. A dosagem de magnésio foi superior à encontrada na Sarcocornia neei $(0,98 \mathrm{~g} / 100 \mathrm{~g})$ e o teor de ferro demonstrou-se semelhante ao encontrado na Sarcocornia perennis (35,02 $\mathrm{mg} / 100 \mathrm{~g})$ e superior à Salicornia ramosissima $(19,67 \mathrm{mg} / 100 \mathrm{~g})$ e à Salicornia europeae (9 mg/100g) (JULIÃO, 2013; RIQUELME et al., 2016).

Já em relação ao zinco, obteve-se resultados semelhantes ao da Sarcocornia neei $(34,73 \mathrm{mg} / 100 \mathrm{~g})$ e maiores que a Salicornia ramosissima $(3,39 \mathrm{mg} / 100 \mathrm{~g})$ e a Sarcocornia perennis $(2,12 \mathrm{mg} / 100 \mathrm{~g})$. Por fim, a dosagem de cobre demonstrou-se superior à Salicornia ramosissima (1,32 mg/100g), à Sarcocornia perennis $(1,12 \mathrm{mg} / 100 \mathrm{~g})$, e à Salicornia herbacea $(1,1 \mathrm{mg} / 100 \mathrm{~g})$ e inferior à Sarcocornia neei (16,95 mg/100g) (JULIÃO, 2013; RIQUELME et al., 2016).

Assim como Bertin et al. (2016) encontrou em seus estudos, variações na análise dos compostos químicos das halófitas podem ocorrer devido ao acúmulo de solutos orgânicos e minerais que são influenciados por sua localização geográfica, forma de cultivo, maturidade e condições de colheita.

Em relação à influência dos tratamentos térmicos na composição química da Sarcocornia ambigua, foram encontradas diferenças significativas $(p<0,05)$ apenas na dosagem de fenóis e de magnésio no tratamento IV, cujos valores demonstraram-se discretamente diminuídos. Visto que as plantas medicinais são sensíveis às temperaturas elevadas, podendo ocorrer alterações qualitativas e quantitativas das substâncias ativas (SILVA et al., 2016), os resultados obtidos indicam que as temperaturas utilizadas no preparo dos alimentos não influenciam na quantificação dos compostos químicos, portanto, não houve perda da qualidade nutricional da planta.

\section{Análise sensorial}

O sabor é considerado um dos fatores mais importantes na escolha dos alimentos e por isso, reduções significativas de sódio levam à diminuição de qualidade sensorial dos alimentos, dificultando a terapêutica dos hipertensos que possuem dieta com restrição ao sal. Para isso, alternativas que possibilitem a redução de sódio são desenvolvidas através da substituição por temperos e especiarias (ALVES, 2014).

Neste estudo, verificou-se a influência da substituição do sal pelo sal verde da Sarcocornia ambigua no tempero da batata frita através da aceitação sensorial do produto alimentício. Participaram da análise 40 julgadores, hipertensos, 70\% do gênero feminino e $30 \%$ do gênero masculino, com idades variando entre 50 e 87 anos. Divididos em faixa etária, a maioria (45\%) representava a idade entre 61 e 70 anos, seguido de $32,5 \%$ entre 71 e 80 anos, $12,5 \%$ entre 50 e 60 anos e $10 \%$ entre 81 e 90 anos.

A Figura 1 indica a frequência de escores no teste de aceitação e a Figura 2 indica a frequência de escores no teste de intenção de consumo. 
Figura 1- Distribuição dos provadores pelos valores hedônicos obtidos no teste de aceitação da batata frita temperada com o sal verde da Sarcocornia ambigua com escala de 9 pontos, variando de 1- desgostei extremamente a 9- gostei extremamente

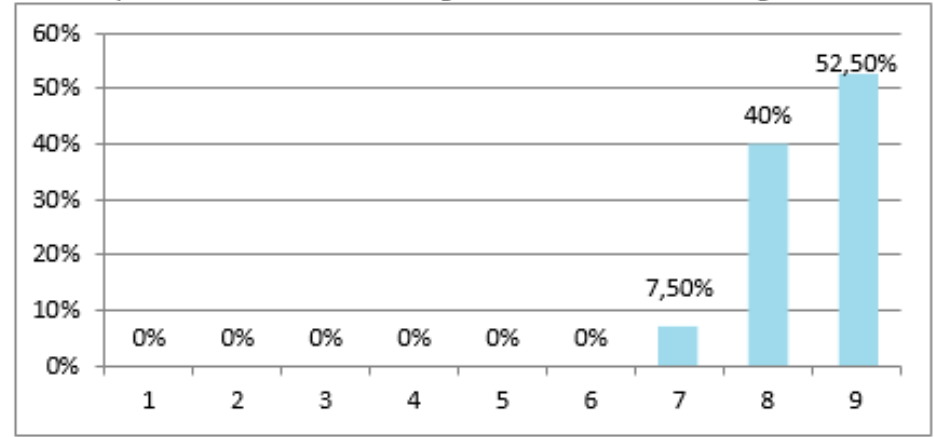

Fonte: As autoras (2017).

Figura 2- Distribuição dos provadores pelos valores hedônicos obtidos no teste de intenção da batata frita temperada com o sal verde da Sarcocomia ambigua com escala de 7 pontos, variando de 1- nunca comeria a 7-comeria sempre

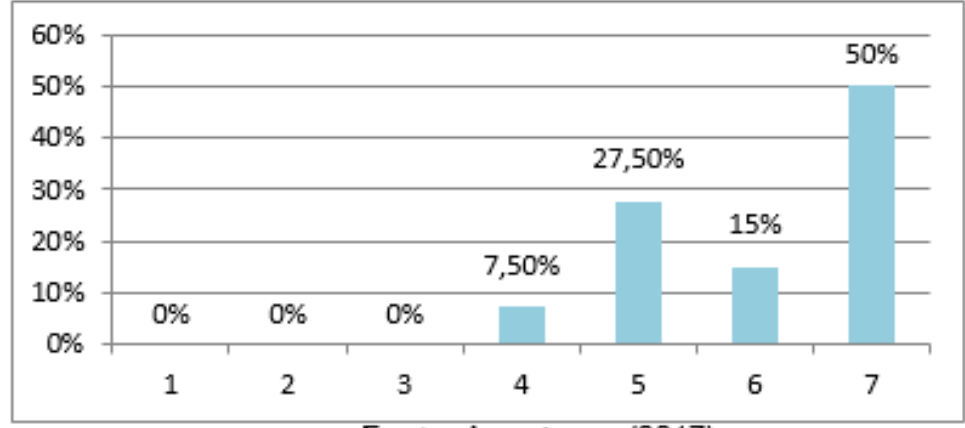

Fonte: As autoras, (2017).

No teste de aceitação, o escore médio encontrado na análise sensorial foi de 8,4 (situando-se próximo ao termo hedônico "gostei muito"). E, para que um produto alimentício seja aceito sensorialmente, tem que possuir média mínima de 7 (DUTCOSKY, 2013). Desse modo, observa-se que a aceitação do produto alimentício preparado com o sal verde da Sarcocornia ambigua teve aceitação significativa.

No teste de intenção, o escore médio encontrado foi de 6 (situando-se no termo hedônico "comeria muito frequentemente"). Esse resultado corrobora com o obtido no teste de aceitação, indicando que, além de possuir ótima aceitação, os provadores demonstram intenção de consumir o produto temperado com o sal verde.

Com resultados semelhantes, Julião (2013) realizou análises sensoriais para avaliar o potencial da Salicornia ramosissima em saladas frescas e em pó, como sal verde, obtendo aceitação significativa em ambos os produtos.

Bezerra (2009) buscou outra alternativa para melhorar a adesão à terapia nutricional de pacientes com restrição ao sódio e avaliou sensorialmente a aceitação do "sal de ervas", composto por orégano, manjericão e alecrim. A aceitabilidade do produto alimentício foi significativa, mostrando-se como uma 
alternativa promissora por ser capaz também de reduzir a quantidade de sódio em até $73 \%$ nos alimentos.

Silvestre, dos Santos e Novello (2016) também verificaram a aceitação sensorial em população de hipertensos, através da avaliação de kibes elaborados com diferentes concentrações de cloreto de sódio $(\mathrm{NaCl})$, cloreto de potássio $(\mathrm{KCl})$ e glutamato monossódico (GM). O estudo obteve aceitação sensorial com redução de até $67 \%$ de sódio no produto alimentício.

Já Alves (2014) avaliou os efeitos da adição dos sais cloreto de potássio e cloreto de magnésio como alternativa de redução de sódio em um cream cheese com adição das culturas probióticas Lactobacillus acidophilus e Bifidobacterium lactis. E, assim como as demais análises sensoriais, encontrou aceitação significativa.

\section{CONSIDERAÇÕES FINAIS}

Os resultados encontrados indicam que a Sarcocornia ambigua não apresentou atividade tóxica pelo teste Allium cepa. Contudo, necessita-se a realização de análises in vivo para aprofundar este estudo.

Em relação à composição química, a planta apresentou elevado valor nutricional, com variedade de nutrientes e compostos bioativos valiosos para uso na indústria alimentícia e farmacêutica. Também foi possível determinar que os tratamentos térmicos não influenciaram a quantificação dos compostos químicos da Sarcocornia ambigua, logo, não houve perda da qualidade nutricional.

Concluindo, o sal verde demonstrou possuir aceitação significativa, com índices ótimos de aceitação e intenção de consumo pelos pacientes hipertensos com restrição ao sódio. Dessa forma, a Sarcocornia ambigua apresentou-se como uma estratégia promissora para atuar como coadjuvante na manutenção da pressão arterial, através da substituição do sal no tempero dos alimentos.

\section{REFERÊNCIAS}

ABREU, L. F.; FARIA, J. D. A. Influência da temperatura e do ácido ascórbico sobre a estabilidade físico-química e atividade enzimática da água de coco (Cocos nucifera L.) acondicionada assepticamente. Food Science and Technology, v. 27, n. 2, p. 226-232, 2007.

AGAWU, E. T. Comparison between Salicornia and Sarcocornia ecotypes to optimize yield for vegetable production applying highly saline irrigation. Dissertação (Mestrado), Ben Gurion University of the Negev, 2012.

ALVES, E. M. V. Efeito da redução do teor de sódio sobre as propriedades físicoquímicas, microbiológicas e sensoriais de cream cheese probiótico. Dissertação (Mestrado), Universidade Estadual de Campinas, 2014.

BERTIN, R. L. et al. Prospecção para exploração da erva de sal (Sarcocornia ambigua-Amaranthaceae): análise histológica, caracterização química, valor nutricional e potencial antioxidante. Tese (Doutorado), Universidade Federal de Santa Catarina, 2014. 
BERTIN, R. L. et al. Mineral composition and bioaccessibility in Sarcocornia ambigua using ICP-MS. Journal of Food Composition and Analysis, v. 47, p. 45$51,2016$.

BEZERRA, M. N. Aceitação do sal de ervas em dieta hipossódica. Monografia (Graduação), Universidade de Brasília, 2009.

BRASIL. Sociedade Brasileira de Cardiologia. VI Diretrizes brasileiras de hipertensão. Arq Bras Cardiol, v. 107, p. 1-83, 2016.

BRASIL. Farmacopéia Brasileira. 5ํㅡㄹ ed. Brasília: Agência Nacional de Vigilância Sanitária, 2010.

CASTRO, D. S. et al. Effect of temperature on the physicochemical composition and bioactive compounds of taro flour obtained in a spouted bed. Brazilian Journal of Food Technology, v. 20, 2017.

DE OLIVEIRA, C. J.; MOREIRA, T. M. M. Caracterização do tratamento nãofarmacológico de idosos portadores de hipertensão arterial. Northeast Network Nursing Journal, v. 11, n. 1, 2016.

DOS SANTOS, B. A. et al. Salt reduction in dry fermented sausages: effects on biochemical, chemical, physical and sensory properties. Tese (Doutorado), Universidade Estadual de Campinas, 2015.

DUTCOSKY, S. D. Análise sensorial de alimentos. 4. ed. Curitiba: Champagnat, 2013.

GARGOURI, M. et al. Cytoprotective and antioxidant effects of the edible halophyte Sarcocornia perennis L. (swampfire) against lead-induced toxicity in renal cells. Ecotoxicology and environmental safety, v. 95, p. 44-51, 2013.

GASTALDON, L. T. et al. Análise sensorial de empadas integrais em crianças na fase escolar. Aliment Nutr, v. 18, n. 3, p. 303-307, 2007.

GIROTTO, E. et al. Adesão ao tratamento farmacológico e não farmacológico e fatores associados na atenção primária da hipertensão arterial. Ciênc Saúde Coletiva, v. 18, n. 6, p. 1763-72, 2013.

GUNNING, D. Cultivating Salicornia europaea (Marsh Samphire). Daithi O'Murchu Marine Research Station \& University College Cork, Dublin, p. 1-50, 2016.

IVANOVA, A. et al. Antioxidant properties of Smilax excelsa. Rivista Italiana delle Sostanze Grasse, v. 83, n. 3, p. 124-128, 2006.

IZEPPI, E. M. Efeitos da densidade de plantio na sobrevivência, desenvolvimento e produção de biomassa da halófita Sarcocornia ambigua (MICHX.) Alonso \& Crespo. Dissertação (Mestrado), Universidade Federal do Rio Grande, 2011. 
JULIÃO, M. R. A. Avaliação do potencial da Salicornia ramosissima para saladas frescas ou em pó (sal verde). Tese (Doutorado), Universidade do Algarve, 2013.

KARADENIZ, F. et al. Effect of Salicornia herbacea on osteoblastogenesis and adipogenesis in vitro. Marine drugs, v. 12, n. 10, p. 5132-5147, 2014.

KIM, S.; KIM, S.; LEE, H. Effect of insoluble dietary fiber extracted from Salicornia herbacea L. on large intestinal function in rats. Korean Journal of Food Science and Technology, v. 46, n. 5, p. 648-654, 2014.

KIM, M. J.; JUN, H. Y.; KIM, J. H. Anti-obesity effect of Korean Hamcho (Salicornia herbacea L.) powder on high-fat diet-induced obese rats. Journal of Nutrition and Health, v. 48, n. 2, p. 123-132, 2015.

KRUGER, R. A. Análise da toxicidade e da genotoxicidade de agrotóxicos utilizados na agricultura utilizando bioensaios com Allium cepa. Univ. Feevale, v. 58, 2009.

LEE, H. Acute oral toxicity of Salicornia herbacea L. extract in mice. Biomedical Science Letters, v. 22, n. 2, p. 46-52, 2016.

MANOUSAKI, E.; KALOGERAKIS, N. Halophytes present new opportunities in phytoremediation of heavy metals and saline soils. Industrial \& Engineering Chemistry Research, v. 50, n. 2, p. 656-660, 2010.

MENDES, G. S.; MORAES, C. F.; GOMES, L. Prevalência de hipertensão arterial sistêmica em idosos no Brasil entre 2006 e 2010. Revista Brasileira de Medicina de Família e Comunidade, v. 32, n. 9, p. 273-278, 2014.

PANTH, N. et al. Protective effect of Salicornia europaea extracts on high salt intake-induced vascular dysfunction and hypertension. International Journal of Molecular Sciences, 17(7), 2016.

PATEL, S. Salicornia: evaluating the halophytic extremophile as a food and a pharmaceutical candidate. 3 Biotech, v. 6, n. 1, p. 1-10, 2016.

PEREIRA, R. M. et al. Estudos iniciais sobre o cultivo da halófita Sarcocornia perennis em Santa Catarina. Monografia (Graduação), Universidade Federal de Santa Catarina, 2012.

PINHEIRO, I. C. Produção da halófita Sarcocornia ambigua e Litopenaeus vannamei em sistema de aquaponia com bioflocos. Tese (Doutorado), Universidade Federal de Santa Catarina, 2015.

PINHEIRO, I. et al. Production of the halophyte Sarcocornia ambigua and pacific white shrimp in an aquaponic system with biofloc technology. Ecological Engineering, v. 100,p. 261-267, 2017.

RIQUELME, J. et al. Nutritional and functional characterization of wild and cultivated Sarcocornia neei grown in Chile. Ciencia e Investigación Agraria, v. 43, n. 2, p. 283-293, 2016. 
ROCHA, M. S. et al. Caracterização físico-química e atividade antioxidante (in vitro) de frutos do cerrado Piauiense. Rev. Bras. Frutic, v. 35, n. 4, p. 933-941, 2013.

RODRIGUES, F. S. M. et al. Benefício da utilização do novo alimento funcional salgante sem sódio sobre os níveis de pressão arterial em animais hipertensos. Revista de Pesquisa e Inovação Farmacêutica, v.5, n. 1, 2015.

SCHVEITZER, B.; SUZUKI, A. Métodos de análise foliar utilizados no Laboratório de Ensaio Químico da Epagri/EECd. Doc. n. 242, ISSN 0100-8986, 2013.

SILVA, F. M. et al. Práticas de vida de portadores de hipertensão arterial. Rev. enferm. UERJ, v. 21, n. 1, p. 54-59, 2013.

SILVA, A. C. B. et al. Qualidade nutricional e físico-química em cenoura (Daucus carota I.) in natura e minimamente processada. DEMETRA: Alimentação, Nutrição \& Saúde, v. 11, n. 2, p. 355-367, 2016.

SILVESTRE, F. K.; DOS SANTOS, E. F.; NOVELLO, D. Kibes com teor reduzido de cloreto de sódio: caracterização físico-química e sensorial entre hipertensos. Multitemas, v. 21, n. 49, 2016.

TOMAZONI, T.; SIVIERO, J. Consumo de potássio de idosos hipertensos participantes do Programa Hiperdia do município de Caxias do Sul, RS. Rev Bras Hipertens, v. 16, n. 4, p. 246-250, 2009.

VENTURA, Y.; SAGI, M. Halophyte crop cultivation: the case for Salicornia and Sarcocornia. Environmental and Experimental Botany, v. 92, p. 144-153, 2013. 